\title{
Wireless Operators in a Shared Spectrum
}

\author{
Mark Felegyhazi and Jean-Pierre Hubaux \\ EPFL - Switzerland \\ email: \{mark.felegyhazi,jean-pierre.hubaux\}@epfl.ch
}

Poster abstract at Mobicom 2005

\section{INTRODUCTION}

Cellular networks are notoriously difficult to design and operate; in particular, defining the optimal location of the base stations and fine tuning their configuration parameters is very challenging. For this reason, government agencies (such as the FCC for the US) usually sell or rent, for example by auction, each operator a frequency band for its exclusive usage in a given country or region. Only a small part of the whole spectrum is allocated as a shared spectrum, in which networks function in the same (unlicensed) frequency band.

With the progress of technology and the fast growing demand for ubiquitous high speed wireless services, it is clear that the pressure towards more flexibility of the usage of the spectrum will only increase. Therefore, the government agencies are likely to adapt the current regulations in order to increase the proportion of the unlicensed spectrum $[1,2]$.

The evolution towards unlicensed frequency bands can lead to a better usage of the spectrum. Yet, it would also create a novel situation, in which the base stations of different operators would interfere with each other. An operator may be tempted to let its base stations transmit at the maximum authorized level. But by doing this, it would maximize interference not only to its own base stations, but also to the base stations of the other operators, and to all mobiles in power range; in addition, it faces the danger that the other operators retaliate by behaving in the same way.

In our work, we assume that mobile users can freely roam across the base stations located in their neighborhood, attaching to the one offering the most favorable signal quality (i.e., the one with the strongest pilot signal), irrespectively of the operator to which the base station belongs. From the interference perspective, this scenario could be much more efficient than the current practice, because it enabled mobile devices to find the "closest" base station in the area and hence both mobile devices and base stations could significantly decrease their transmission power.

To mitigate interference, the shared frequency band is usually split up into channels (i.e., separated frequency sub-bands), but the pilot signal is typically emitted on a single shared channel for all the base stations, which results in mutual interference of the pilot signals (in CDMA networks, the interference of the pilot signals is

Permission to make digital or hard copies of all or part of this work for personal or classroom use is granted without fee provided that copies are not made or distributed for profit or commercial advantage and that copies bear this notice and the full citation on the first page. To copy otherwise, to republish, to post on servers or to redistribute to lists, requires prior specific permission and/or a fee.

Mobicom '05 Köln, Germany

Copyright 2005 ACM X-XXXXX-XX-X/XX/XX ...\$5.00. referred to as the pilot pollution [4]).

In our scenario, we also assume that the operators want to cover the largest possible area by increasing the transmission range of their base stations. At the same time, they want to minimize the interference. We assume that this goal corresponds to their motivation for maximizing the number of users who attach to their base stations. We model this situation as a game in terms of power control of the base stations.

Recently, the coexistence of wireless operators in a non-shared spectrum was addressed in two contributions. Halldórsson et al. [3] study channel assignment strategies for Wi-Fi operators. They use the maximum graph coloring problem to identify Nash equilibria and they also provide a bound on the price of anarchy of these equilibria. Zemlianov and de Veciana [6] consider the scenario, in which users are able to choose between a cellular network and a Wi-Fi network. They show that congestion sensitive strategies are better than proximity-based strategies. None of these works considers the power control of the base stations.

The remainder is organized in the following way. In Section 2, we describe the system model and the corresponding power control game. We summarize our results in Section 3. Finally, we conclude in Section 4.

\section{MODEL}

In our work, we assume a wireless communication network with two operators $A$ and $B$ and several users. Base stations and user devices operate on the same unlicensed band of the frequency spectrum. We denote the set of base stations that belong to player $i \in\{A, B\}$ by $\mathcal{B}_{i}$. Base station transmit pilot signals to notify users about their presence. We assume that the expected position of users is uniformly distributed over a given area and they attach to the base station with the strongest received pilot signal. Hence the goal of the operators is the maximize the area, in which their pilot signal is the strongest. We apply a radio range model that is widely used in the literature (for example the service coverage defined in [5]), meaning that we model the coverage of the base stations without interference with a circle. To further specify our model, we assume that: (i) Operators want to provide wireless access service everywhere. Thus, no place remains uncovered in the area. (ii) There exists a limitation $R_{M A X}$ defined by the regulator of the wireless telecommunication system. Furthermore, we denote the smallest common radio range which enables the base stations to cover the whole are by $R_{M I N}=\max _{k} R_{k, M I N}$, where $k \in\left\{\mathcal{B}_{A}, \mathcal{B}_{B}\right\}$. Figure 1 shows an example for the coverage of a base station.

We model the power control problem with two operators as a two-player, nonzero-sum game. The strategy of the players defines their best radio range. The goal of the players is to maximize the 


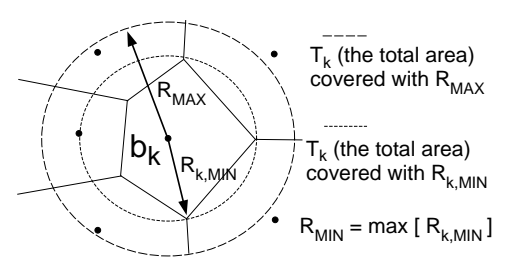

Figure 1: Ranges in a general topology of base stations. $R_{M A X}$ is defined by the regulator and $R_{k, M I N}$ is the smallest range with which they can cover the whole space around base station $b_{k} . R_{M I N}$ is the largest of these minimum ranges $R_{k, M I N}$ (meaning that it is the smallest common radio range which enables full coverage).

area they cover with their pilot signal while minimizing the interference in the network. This goal is expressed in their utility function. We define the utility of player $i$ as a function of the coverage area $\left(O_{k}\right)$ of its base stations $b_{k}$ as follows:

$$
U_{i}=\sum_{b_{k}=1}^{\left|\mathcal{B}_{i}\right|}\left(1+\gamma_{i}\right) \cdot O_{k}-\gamma_{i} \cdot r_{k}^{2} \cdot \pi
$$

where $\gamma_{i}$ is the sensitivity of player $i$ to interference, $O_{k}$ is the coverage area and $r_{k}$ is the radio range of base station $b_{k} \in \mathcal{B}_{i}$.

\section{RESULTS}

We first present a simplified scenario and derive the principles of cooperation in it. Then we extend our study to more complex scenarios.

To prove stability points in the power control game, we rely on the following game-theoretic concepts.

DEFINITION 1. In a Nash equilibrium, none of the players can unilaterally change its strategy to increase its utility.

Furthermore, if several Nash-equilibria exist, the concept of Paretooptimality is used to chose the best one.

DEFINITION 2. A set of strategies is Pareto-optimal (or socially optimal), if none of the players can increase its utility unless the utility of another player decreases.

Note that a Pareto-optimal set of strategies does not necessarily constitute a Nash-equilibrium.

Consider the following simple scenario called the grid scenario. Assume that the base stations are placed alternately on the vertices of a grid and the radio ranges can be set only once (the latter assumption defines a single-stage game). Let us also assume that the players choose the same radio range for all of their base stations (an example is shown in Figure 2). In this scenario, several Nashequilibria exist depending on the sensitivity values of the players. We state the best Nash-equilibria in the following theorem.

THEOREM 1. Depending on the sensitivity values $\gamma_{i}$, three Paretooptimal Nash-equilibria exist in the grid scenario: (i) both players play the radio range $R_{M A X}$ or (ii) $R_{M I N}$ or (iii) one player plays the radio range equal to zero (its utility is maximized by not playing), while the other covers the whole area.

If the players can set different radio ranges for their base stations then we prove the following theorem.

THEOREM 2. Finding Nash equilibria in the power control game for general values of radio ranges is NP-complete.

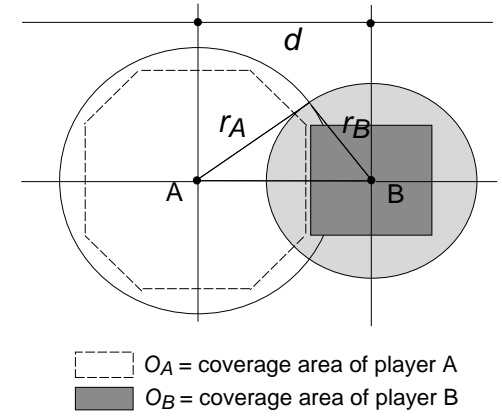

Figure 2: Coverage areas of a base stations on the grid, illustrated with two base stations.

Assuming there is a general topology of base station within the network of a player using a common radio range, we show that cooperation (playing $R_{M I N}$ ) can be enforced using punishments.

THEOREM 3. If both players play a strategy based on punishments then it results in a Nash equilibrium.

We emphasize that Theorem 1 applies only for the grid scenario with two players, Theorems 2 and 3 apply for a general scenario with arbitrary number of players.

\section{CONCLUSION}

In this work, we have investigated the problem of co-existing wireless operators in a shared spectrum. We have assumed that the operators apply power control of the pilot signal at the base stations to mitigate interference, while providing a permanent service to the users.

The contribution of this paper is threefold. First, we have shown that Nash equilibria exist if the operators set the power of their base stations at the beginning of the operation of the network. We have identified different equilibrium situations depending on the sensitivity of the operators to interference. Second, we have shown that the solution of the power control problem is NP-complete for a general topology of base stations and a general set of radio ranges. Third, we have proved a condition for which a socially optimal Nash equilibrium exists and that it can be enforced using punishments. This result holds in the general topology of base stations with specific radio ranges. In general, our results show which operation points are beneficial for the players and how these should be achieved.

\section{REFERENCES}

[1] Y. Benkler. Some economics of wireless communication. Harvard Journal of Law and Technology, 16(1), Fall 2004.

[2] G. Faulhaber and D. Farber. Spectrum management: Property rights, markets, and the commons. In Telecommunications Policy Research Conference Proceedings, 2003.

[3] M. M. Halldórsson, J. Y. Halpern, L. (Erran) Li, and V. S. Mirrokni. On spectrum sharing games. In Proceedings of PODC 2004, pages 107-114, July 25-28 2004.

[4] T. S. Rappaport. Wireless Communications: Principles and Practice (2nd Edition). Prentice Hall, 2002.

[5] M. Schwartz. Mobile Wireless Communications. Cambridge Univ. Press, 2005.

[6] A. Zemlianov and G. de Veciana. Cooperation and decision making in wireless multi-provider setting. In Proceedings INFOCOM 2005, March 13-17 2005. 